\title{
Oxygen-Storage Behavior and Local Structure in Ti-substituted $\mathrm{YMnO}_{3}$
}

\author{
I. Levin ${ }^{1}$, V. Krayzman ${ }^{1}$, T. A. Vanderah ${ }^{1}$, M. Tomczyk ${ }^{2}$, H. Wu ${ }^{1}$, M. G. Tucker ${ }^{3}$, H. Y. Playford ${ }^{4}$, J. C. \\ Woicik $^{1}$, C. L. Dennis ${ }^{1}$, and P. M. Vilarinho ${ }^{2}$ \\ ${ }^{1}$ National Institute of Standards and Technology \\ Gaithersburg MD 20899, USA \\ ${ }^{2}$ Department of Ceramics and Glass Engineering, University of Aveiro, \\ Aveiro, 3810-193 Portugal \\ ${ }^{3}$ Spallation Neutron Source, Oak Ridge National Laboratory, \\ Oak Ridge, TN, USA \\ ${ }^{4}$ ISIS Facility, Rutherford Appleton Laboratory, Didcot, OX, UK
}

\begin{abstract}
Hexagonal manganates $\mathrm{RMnO}_{3}(\mathrm{R}=\mathrm{Y}, \mathrm{Ho}$, Dy) have been recently shown to exhibit oxygenstorage capacities promising for three-way catalysts, air-separation, and related technologies. Here, we demonstrate that Ti substitution for $\mathrm{Mn}$ can be used to chemically tune the oxygenbreathing properties of these materials towards practical applications. Specifically, $\mathrm{Y}\left(\mathrm{Mn}_{1-\mathrm{x}} \mathrm{Ti}_{\mathrm{x}}\right) \mathrm{O}_{3}$ solid solutions exhibit facile oxygen absorption/desorption via reversible $\mathrm{Ti}^{3+} \leftrightarrow \mathrm{Ti}^{4+}$ and $\mathrm{Mn}^{3+} \leftrightarrow \mathrm{Mn}^{4+}$ reactions already in ambient air at $\approx 400{ }^{\circ} \mathrm{C}$ and $\approx 250{ }^{\circ} \mathrm{C}$, respectively. On cooling, the oxidation of both cations is accompanied by oxygen uptake yielding a formula $\mathrm{YMn}^{3+}{ }_{1-\mathrm{x}-}$ ${ }_{y} \mathrm{Mn}^{4+}{ }_{y} \mathrm{Ti}^{4+}{ }_{x} \mathrm{O}_{3+\delta}$. The presence of $\mathrm{Ti}$ promotes the oxidation of $\mathrm{Mn}^{3+}$ to $\mathrm{Mn}^{4+}$, which is almost negligible for $\mathrm{YMnO}_{3}$ in air, thereby increasing the uptake of oxygen beyond that required for a given $\mathrm{Ti}^{4+}$ concentration. The reversibility of the redox reactions is limited by sluggish kinetics; however, the oxidation process continues, if slowly, even at room temperature. The extra oxygen atoms are accommodated by the large interstices within a triangular lattice formed by the $\left[\mathrm{MnO}_{5}\right]$ trigonal bipyramids. According to bond distances from Rietveld refinements using the neutron diffraction data, the $\mathrm{YMnO}_{3}$ structure features under-bonded $\mathrm{Mn}$ and even more severely under-bonded oxygen atoms that form the trigonal bases of the $\left[\mathrm{MnO}_{5}\right]$ bipyramids. The tensile bond strain around the 5-fold coordinated $\mathrm{Mn}$ site and the strong preference of $\mathrm{Ti}^{4+}$ (and $\mathrm{Mn}^{4+}$ ) for higher coordination numbers likely provide driving forces for the oxidation reaction. Reverse Monte Carlo refinements of the local atomic displacements using neutron total scattering revealed how the excess oxygen atoms are accommodated in the structure by correlated local displacements of the host atoms. Large displacements of the under-bonded host oxygen atoms play a key part in this lattice-relaxation process, facilitating reversible exchange of significant amounts of oxygen with atmosphere.
\end{abstract}




\section{Introduction}

$\mathrm{YMnO}_{3}$, which crystallizes with a hexagonal structure [1], has been studied extensively as an example of a non-perovskite multiferroic [e.g., 2-8]. This structure consists of layers of vertexsharing trigonal $\left[\mathrm{MnO}_{5}\right]$ bi-pyramids linked by layers of 8-fold coordinated $\mathrm{Y}^{3+}$ ions (Fig. 1). On cooling, at $\mathrm{T} \approx 1000{ }^{\circ} \mathrm{C}, \mathrm{YMnO}_{3}$ undergoes a phase transition from the high-temperature $P 6_{3} / \mathrm{mmc}$ form to the non-centrosymmetric $P 6_{3} \mathrm{~cm}$ polymorph, manifested in rotations of $\left[\mathrm{MnO}_{5}\right]$ polyhedra with concurrent ferrielectric-like $Y$ displacements along the $c$-axis [7]. The effective coordination of $Y$ changes from 8-fold to $(7+1)$-fold because of the strong off-centering of $Y$ atoms within the -O-Y-O- chains along the $c$-axis. The transition is accompanied by loss of the inversion center and tripling of the unit-cell volume. Despite numerous experimental and theoretical studies [2-8], the exact origin of ferroelectricity and even the actual Curie temperature in this compound remain debatable.

The point defect chemistry in $\mathrm{YMnO}_{3}$ is also somewhat uncertain. Two conflicting scenarios that propose mixed $3+/ 2+[9]$ and $3+/ 4+[10]$ oxidation states for $\mathrm{Mn}$, respectively, have been proposed from the variable-temperature dielectric and electrical-conductivity studies. Thermal gravimetric analysis (TGA) revealed that $\mathrm{YMnO}_{3}$ remains nearly stoichiometric if heat-treated in air but undergoes oxidation to $\mathrm{YMnO}_{3+\delta}$ with $\delta=0.13$, upon thermal cycling in oxygen [11-13]. The oxidation occurs between $300{ }^{\circ} \mathrm{C}$ and $200{ }^{\circ} \mathrm{C}$. A much larger oxygen excess, $\delta=0.38$, has been achieved using an oxygen pressure of 190 bar [11]. Similar behavior was reported for a series of hexagonal $\mathrm{RMnO}_{3}(\mathrm{R}=\mathrm{Ho}, \mathrm{Er}, \mathrm{Dy}, \mathrm{Y})$ compounds $[12,13]$. For a given set of conditions, the amount of excess oxygen increased markedly with increasing ionic radius of the $\mathrm{R}$ species. The largest value of oxygen-storage capacity reported for the $\mathrm{RMnO}_{3}$ materials under 1 bar oxygen pressure is $\approx 1200 \mu \mathrm{mol} \cdot 0 / \mathrm{g}$ at $\approx 350{ }^{\circ} \mathrm{C}$ [11], which, combined with relatively low oxygen absorption/desorption temperatures, renders them potentially viable for air-separation and related applications. Reportedly [12], the $P 6_{3} \mathrm{~cm}$ structure is preserved for $\delta<0.13$, while for higher oxygen content the symmetry is reduced first to trigonal $R 3 c$ (e.g., $\delta \approx 0.28$ ) and then to orthorhombic $\mathrm{Pca}_{1}$ (e.g., $\delta=0.4$ ). All these structures can be derived from the archetypical $\mathrm{PG}_{3} / \mathrm{mmc}$ symmetry by imposing different patterns of rotations for the $\left[\mathrm{MnO}_{5}\right]$ bipyramids in consecutive $c$-layers.

In the present work, we show that while $\mathrm{YMnO}_{3}$ indeed remains mostly stoichiometric in air at least up to $1150{ }^{\circ} \mathrm{C}, \mathrm{YMn}_{1-x} \mathrm{Ti}_{\mathrm{x}} \mathrm{O}_{3+\delta}$ solid solutions rapidly absorb atmospheric oxygen on cooling below $400{ }^{\circ} \mathrm{C}$. This enhanced oxygen absorption relative to $\mathrm{YMnO}_{3}$ is attributed to the redox reactions involving both $\mathrm{Ti}$ and $\mathrm{Mn}$; importantly, the presence of $\mathrm{Ti}^{4+}$ promotes partial oxidation of $\mathrm{Mn}^{3+}$ to $\mathrm{Mn}^{4+}$. We use neutron total scattering to establish the local structure in pure and Tisubstituted $\mathrm{YMnO}_{3}$ and demonstrate how locally-correlated atomic shifts accommodate the

excess oxygen atoms in the interstices of the host lattice. Our results suggest that substitutions on the $\mathrm{Mn}$ sites can be effective in optimizing the oxygen-storage properties of hexagonal $\mathrm{RMnO}_{3}$ materials for practical use. 


\section{Experimental}

Ceramic $\mathrm{YMn}_{1-\mathrm{x}} \mathrm{Ti}_{\mathrm{x}} \mathrm{O}_{3}(x=0,0.05,0.125)$ samples were processed using conventional solid-state methods as detailed in [14]. "As-prepared" samples had been sintered in air at $1400{ }^{\circ} \mathrm{C}$ for 5 hours. Previous studies of this system [14] indicated that for $x<0.175$, the solid solutions retain the $P 6_{3} \mathrm{~cm}$ symmetry of the end compound [11]. Indeed, all our samples were phase pure with $X$-ray diffraction (XRD) patterns indexable according to the $P 6_{3} \mathrm{Cm}$ structure. Thus, the aliovalent substitution of $\mathrm{Ti}^{4+}$ for $\mathrm{Mn}^{3+}$ in $\mathrm{YMnO}_{3}$ requires charge compensation which must occur by one or more of the following mechanisms: 1) reduction of $\mathrm{Mn}^{3+}$ to $\mathrm{Mn}^{2+}, 2$ ) reduction of $\mathrm{Ti}^{4+}$ to $\mathrm{Ti}^{3+}$, 3 ) addition of oxygen above 3 atoms per formula unit, and 4) formation of cation vacancies. As we show below, the structure absorbs excess oxygen to provide charge neutrality.

Thermal analyses were carried out between room temperature and $1150{ }^{\circ} \mathrm{C}$ with a TA [15] instruments SDT Q600 simultaneous TGA/DSC in platinum sample cups under flowing air or helium (100 ml/min), with heating/cooling rates of $5{ }^{\circ} \mathrm{C} / \mathrm{min}$ and sample sizes $100-150 \mathrm{mg}$. The $\mathrm{YMnO}_{3}$ sample was additionally measured in oxygen $(100 \mathrm{ml} / \mathrm{min})$

Variable-temperature XRD was performed using a Panalytical Xpert Pro diffractometer equipped with an incident beam monochromator, a position-sensitive Pixcel detector, and an Anton Paar XRK2000 temperature stage. The sample powder was dispersed in ethanol and deposited as a thin layer on the platinum heater. The measurements were performed in air, helium, and nitrogen (1 atm).

Samples for transmission electron microscopy (TEM) were prepared either using mechanical thinning followed by conventional ion-milling until perforation or by grinding the powder in ethanol and dispersing the suspension on lacey-carbon-coated copper grids. The samples were examined in an FEI Titan TEM (300 kV) equipped with a high-angle annular dark field detector and a Gatan Enfina electron energy-loss spectrometer.

Variable field (from $+5570 \mathrm{kA} / \mathrm{m}=70000$ Oe to $-5570 \mathrm{kA} / \mathrm{m}$ and back) and temperature (from 300 to $950 \mathrm{~K}$ ) magnetic measurements were performed on sintered pellets using a Quantum Design superconducting quantum interference device (SQUID) vibrating-sample magnetometer (VSM) equipped with a sample oven.

Neutron powder diffraction (NPD) measurements were performed for $\mathrm{YMnO}_{3}$ and $\mathrm{YMn}_{0.875} \mathrm{Ti}_{0.125} \mathrm{O}_{3}$ samples using BT-1 (constant wavelength $\lambda=1.5405 \AA$ ) and Polaris (time of flight) instruments at NIST and ISIS, respectively. The sample powder was loaded in vanadium containers and temperature control was achieved using furnaces. The measurements with BT-1 were performed on heating $\left(1^{\text {st }}\right.$ heating cycle) at six temperatures between $25^{\circ} \mathrm{C}$ and $950{ }^{\circ} \mathrm{C}$ in vacuum. On Polaris, the sample was first measured on heating in $400 \mathrm{mTorr}$ helium (this was the maximum pressure compliant with the facility's safety regulations) at a series of 
temperatures between $25^{\circ} \mathrm{C}$ and $1000{ }^{\circ} \mathrm{C}$, then cooled under the same atmosphere to $200{ }^{\circ} \mathrm{C}$, and measured again upon heating in vacuum [16] between $200^{\circ} \mathrm{C}$ and $600{ }^{\circ} \mathrm{C}$.

$\mathrm{X}$-ray absorption fine structure (XAFS) was measured for the $\mathrm{Mn}$ K-edge in $\mathrm{YMnO}_{3}$ and $\mathrm{YMn}_{0.875} \mathrm{Ti}_{0.125} \mathrm{O}_{3}$ samples using the NIST X23A2 beamline at the National Synchrotron Light Source. The measurements were performed in transmission. Temperature control was achieved using the Linkam THMS600 stage. Transmission data for a Mn foil positioned downstream from the sample was recorded simultaneously with each scan for energy calibration. XAFS data were processed using the Athena software [17].

Rietveld refinements using the XRD data and NPD data were performed in GSAS. Neutron total scattering patterns were processed in GUDRUN to obtain the scattering $S(Q)$ and pairdistribution $G(r)$ functions ( $Q_{\max } \approx 30 \AA^{-1}$ was used in the Fourier transform). The $G(r)$ was initially analyzed using a crystallographic Rietveld-derived model in the PDFGUI software [18]. Structural refinements using $S(Q), G(r)$, and neutron Bragg profile were performed in RMCProfile [19]. An atomic configuration contained $11 \times 11 \times 6$ unit cells with a total of 21,780 atoms. For each structure, the refinements were repeated five times and the structural characteristics of interest were obtained by averaging over the five refined configurations, which represent equivalent but different snapshots of the atomic arrangements.

\section{Results and Discussion}

\subsection{Thermal Analysis: Oxygen Absorption/Desorption}

$\mathrm{YMnO}_{3}$ exhibits a barely detectable change in mass at $\approx 250{ }^{\circ} \mathrm{C}$ upon thermal cycling in air (Fig. $2 a)$. This change, which becomes more noticeable in oxygen, has been attributed to partial oxidation of $\mathrm{Mn}^{3+}$ to $\mathrm{Mn}^{4+}$ (on cooling) yielding excess oxygen in the sample with a chargecompensated formula $\mathrm{YMnO}_{3+\delta}$ [11-13]. The loss of this small amount of excess oxygen was observed between $250{ }^{\circ} \mathrm{C}$ and $300{ }^{\circ} \mathrm{C}$ in the $\mathrm{TGA}$ of $\mathrm{YMnO}_{3}$ under helium. (These measurements additionally ruled out the presence of any significant amount of $\mathrm{Mn}^{2+}$, in accord with the behavior of the lattice parameters presented in the next section.) The behavior of $\mathrm{YMn}_{0.875} \mathrm{Ti}_{0.125} \mathrm{O}_{3+\delta}$ is markedly different. In air (Fig. 2b), the first heating cycle was consistently unique with the onset of an endothermic effect near $350^{\circ} \mathrm{C}$ accompanied first by a slight gain in mass followed by a significant mass loss just near $400{ }^{\circ} \mathrm{C}$; after cooling, an overall $0.3 \%-0.4 \%$ mass loss was observed. At high temperatures the TGA curve indicated continuous mass loss with no plateau up to $1150{ }^{\circ} \mathrm{C}$. The second and subsequent heating cycles (performed immediately one after the other) were very similar to each other with a negligible overall change after cooling; however, the onset of an endothermic process consistently occurred near $375{ }^{\circ} \mathrm{C}$ and was accompanied by a well-defined mass increase $(0.1 \%$ to $0.2 \%)$ which reaches a maximum near $410{ }^{\circ} \mathrm{C}$ and then continuously decreases, with an overall mass loss of $0.5 \%$ at $1150^{\circ} \mathrm{C}$. Re-analysis of a residue of the same sample left for 8 months in air demonstrated that 
the unique first cycle was again observed, indicating that the sample had slowly re-oxygenated to the apparently equilibrium composition. Further experiments, which involved soaking the sample during the second cycle at $360^{\circ} \mathrm{C}$ for 16 hours prior to cooling it to room temperature, revealed significant mass increase over time. The TGA/DSC curves recorded upon subsequent heating and cooling of this sample (i.e. the third cycle) were similar to those characteristic for the first cycle. These results confirm that the overall mass loss observed after the first cycle as well as the absence of a peak at $375{ }^{\circ} \mathrm{C}$ on cooling for the subsequent cycles are determined by slow kinetics of oxygenation below $400^{\circ} \mathrm{C}$.

In helium (Fig. 3a), $\mathrm{YMn}_{0.875} \mathrm{Ti}_{0.125} \mathrm{O}_{3 \delta}$ undergoes abrupt mass loss near $325{ }^{\circ} \mathrm{C}$ followed by gradual loss above $450^{\circ} \mathrm{C}$ which continues up to $1150{ }^{\circ} \mathrm{C}$ with no plateau observed. On cooling, the mass remains essentially constant with an overall loss of $1.1 \%$ at room temperature. No detectable change in mass is observed during the subsequent heating cycle. The same sample cycled again in air (Fig. 3b) exhibits an onset of an exothermic event near $200^{\circ} \mathrm{C}$ accompanied by increase in mass of $0.95 \%$ at $400{ }^{\circ} \mathrm{C}$, following by an endothermic process with a marked mass loss just above $400{ }^{\circ} \mathrm{C}$ and then a gradual decrease in mass up to $1150{ }^{\circ} \mathrm{C}$. For $\mathrm{YMn}_{0.95} \mathrm{Ti}_{0.05} \mathrm{O}_{3}$ subjected to the same heating cycle in helium, the mass loss at room temperature is $0.44 \%$; that is, the loss scales with the Ti content.

Assuming fully occupied $\mathrm{Y}$ sites and nominal $3^{+}$and $4^{+}$oxidation states for $\mathrm{Mn}$ and $\mathrm{Ti}$, respectively, the charge-compensated formula would be $\mathrm{YMn}_{0.875} \mathrm{Ti}_{0.125} \mathrm{O}_{3.0625}$ with a formula mass $\mathrm{FW}=191.9569 \mathrm{~g} / \mathrm{mol}$. The change in $\mathrm{FW}$ relative to $\mathrm{YMn}_{0.875} \mathrm{Ti}_{0.125} \mathrm{O}_{3}$ (i.e., no extra oxygen, $\mathrm{Ti}^{3+}$ ) of $0.5 \%$ is only about half of the $1.1 \%$ mass loss observed by TGA under helium. Assuming that the product of reduction in helium at $1150{ }^{\circ} \mathrm{C}$ is stoichiometric $\mathrm{YMn}_{0.875} \mathrm{Ti}_{0.125} \mathrm{O}_{3}$ (all $\left.\mathrm{Mn}^{3+} / \mathrm{Ti}^{3+}\right)$, our results indicate that the oxygen content of the equilibrium sample is higher than that required to charge-compensate the added $x=0.125 \mathrm{~mol} \mathrm{Ti}^{4+}$. This indicates partial oxidation of $\mathrm{Mn}^{3+}$ to $\mathrm{Mn}^{4+}$, and an equilibrium formula of $\mathrm{YMn}^{3+}{ }_{0.74} \mathrm{Mn}^{4}{ }_{0.135} \mathrm{Ti}^{4+}{ }_{0.125} \mathrm{O}_{3.13}$, with $\approx 15 \%$ of the $\mathrm{Mn}$ oxidized to $4+$. The unique first TGA cycle in air is the result of slow re-oxidation kinetics, with the observed mass upon cooling indicating that much less of the $\mathrm{Mn}$ is present as 4+. Our results on $\mathrm{YMnO}_{3}$ and $\mathrm{YMn}_{0.875} \mathrm{Ti}_{0.125} \mathrm{O}_{3+\delta}$ combined suggest that the $\mathrm{Ti}^{3+} \rightarrow \mathrm{Ti}^{4+}$ and $\mathrm{Mn}^{3+} \rightarrow \mathrm{Mn}^{4+}$ reactions occur (on cooling) around $\approx 400{ }^{\circ} \mathrm{C}$ and $\approx 250{ }^{\circ} \mathrm{C}$, respectively. The general formula of the Ti-substituted $\mathrm{YMnO}_{3}$ prepared and annealed in air is $\mathrm{YMn}^{3+}{ }_{1-x-y} \mathrm{Mn}^{4+}{ }_{y} \mathrm{Ti}^{4+}{ }_{x} \mathrm{O}_{3+\delta}$, with the value of $\delta$ determined by the Ti content and the fraction of the $\mathrm{Mn}^{3+}$ that oxidizes to the $4+$ state.

The $\mathrm{Mn}^{4+} \rightarrow \mathrm{Mn}^{3+}$ reaction on heating is further supported by the behavior of the $\mathrm{Mn} K$ edge in the X-ray absorption spectra: upon the first heating of the as-prepared $x=0.125$ sample, the main edge shifts abruptly to lower energies between $200{ }^{\circ} \mathrm{C}$ and $300{ }^{\circ} \mathrm{C}$, as expected for a change from the mixed $\left(\mathrm{Mn}^{3+}+\mathrm{M}^{4+}\right)$ to $\mathrm{Mn}^{3+}$ oxidation state (Fig. 4). This anomaly disappears on the second heating, which is consistent with the TGA results, suggesting that the $\mathrm{Mn}^{3+} \rightarrow \mathrm{Mn}^{4+}$ reaction is kinetically hindered at room temperature. Conceivably, re-formation of $\mathrm{Mn}^{4+}$ is hindered owing to the unfavorable 5 -fold coordination site. 
We observed that the oxidation behavior of the $\mathrm{Y}(\mathrm{Mn}, \mathrm{Ti}) \mathrm{O}_{3}$ samples was strongly affected by detailed thermal history, heating/cooling rates, etc. Therefore, the present TGA results, while illustrating general oxidation behavior of the Ti-substituted samples, do not reflect detailed equilibrium oxygen concentrations, determination of which requires a separate study.

\subsection{X-ray and neutron powder diffraction}

As indicated in the experimental section, the room-temperature XRD and NPD patterns of $\mathrm{YMn}_{1}$ ${ }_{x} \mathrm{Ti}_{x} \mathrm{O}_{3}(x=0,0.05,0.125)$ are all consistent with the previously reported $P \sigma_{3} \mathrm{~cm}$ structure. However, for $x=0.125$, the superlattice reflections associated with tripling of the unit-cell volume are missing and the patterns (Fig. 5a) can be accounted for by the unit cell of the hightemperature $\mathrm{PG}_{3} / \mathrm{mmc}$ polymorph of $\mathrm{YMnO}_{3}$. Additionally, for these compositions, the Bragg peaks that depend strongly on the $c$ lattice parameter exhibit significant asymmetric broadening. In contrast to the powder diffraction data, electron diffraction patterns from the $\mathrm{YMn}_{0.875} \mathrm{Ti}_{0.125} \mathrm{O}_{3+\delta}$ samples contain sharp superlattice reflections of the $P \sigma_{3} \mathrm{~cm}$ structure and the high-angle annular dark field images display an associated puckering of the $\mathrm{Y}$ layers.

Variable-temperature XRD and NPD measurements in air (Fig. 5a) revealed that the first heating of the as-prepared $\mathrm{YMn}_{0.875} \mathrm{Ti}_{0.125} \mathrm{O}_{3+\delta}$ samples to $\mathrm{T}>300{ }^{\circ} \mathrm{C}$ induces the appearance of superlattice reflections which persist up to $\approx 800^{\circ} \mathrm{C}$ before disappearing at higher temperatures. These superlattice reflections reappear on cooling, and albeit weak, remain visible at roomtemperature. On the second heating (Fig. 5b), the superlattice peaks disappear again between $300{ }^{\circ} \mathrm{C}$ and $400{ }^{\circ} \mathrm{C}$, reappear near $\approx 500{ }^{\circ} \mathrm{C}$, and remain visible up to $\approx 800{ }^{\circ} \mathrm{C}$. This behavior is consistent with the TGA data, which suggests that the absence of the superlattice reflections in the room-temperature diffraction patterns of the as-prepared samples is related to the excess oxygen absorbed by the sample while left on the bench. Presumably, the extra oxygen atoms introduce structural disorder, which destroys the $\mathrm{P}_{3} \mathrm{~cm}$ superlattice. On the second heating, an uptake of oxygen between $300{ }^{\circ} \mathrm{C}$ and $400{ }^{\circ} \mathrm{C}$ is also sufficient to cause such a disorder, as manifested in the disappearance of the superlattice peaks in this temperature range. Thinning the sample for TEM and/or exposure to electron beam in vacuum cause oxygen desorption with re-appearance of the superstructure; thus, unfortunately, conventional TEM/STEM cannot be used to study the samples with excess oxygen.

The lattice parameters obtained for $\mathrm{YMnO}_{3}$ exhibit no discernable anomalies upon heating/cooling, regardless of atmosphere (Fig. 6). For the $\mathrm{YMn}_{1-\mathrm{x}} \mathrm{Ti}_{\mathrm{x}} \mathrm{O}_{3}$ solid solutions with $x=0.05$, the $a$-axis varies nearly linearly with temperature and expands on heating (Fig. 6, Fig. 7) as it does in $\mathrm{YMnO}_{3}$, whereas for $x=0.125$, a weak anomaly is observed near $400{ }^{\circ} \mathrm{C}$. The $c$-axis features anomalous temperature dependence for the solid-solution samples (Fig. 6, Fig. 7). One anomaly occurs around $400{ }^{\circ} \mathrm{C}$, while for $x=0.125$, an additional anomaly is observed near $\approx 900$ ${ }^{\circ} \mathrm{C}$. The first anomaly, the character of which depends strongly on atmosphere, correlates with the onset of major oxygen loss observed by TGA. The high-temperature anomaly coincides with 
the $P \sigma_{3} \mathrm{~cm} \leftrightarrow P \sigma_{3} / \mathrm{mmc}$ phase transition. For $\mathrm{T}<500{ }^{\circ} \mathrm{C}$, the dependence $c(\mathrm{~T})$ changes markedly between the first and second heating cycles in air, which correlates with the difference in the corresponding TGA traces Despite the complex behavior of the lattice parameters, the unit-cell volume for the solid-solution samples varies approximately linearly with temperature. Oxygen absorption is accompanied by expansion of the $a$ - and contraction of the $c$-lattice parameter with a small contraction of the unit-cell volume.

\section{Structure of $\mathrm{YMnO}_{3}$ : Average vs Local}

Rietveld refinements of the $\mathrm{PG}_{3} \mathrm{~cm}$ structure (BT-1 data) for $\mathrm{YMnO}_{3}$ (Fig. 8) yield acceptable agreement factors $\left(R_{\mathrm{wp}}=5.44 \%, \chi^{2}=3.29\right)$; however, the quality of the fit is somewhat inferior to that expected for a fully adequate structural model. The misfit between the experimental and calculated data is manifested primarily in the different distribution of intensities among the overlapping reflections. The fit could not be improved by introducing anisotropic atomic displacement parameters (ADPs). However, for the elevated temperatures, using the anisotropic ADPs improved the fit considerably, lowering the goodness-of-fit by a factor of $\approx 2$, with larger improvements observed at higher temperatures (Fig. 9). Similar results were obtained using the BT-1 and Polaris data.

Bond-valence-sum (BVS) calculations [20] for the room-temperature model yield the following values: $\mathrm{Mn}-2.8$ v.u., Y1 -3.3 v.u., Y2 - 3.0 v.u., $01-2.1$ v.u., $\mathrm{O} 2-2.2$ v.u., $\mathrm{O} 3-1.65$ v.u., $\mathrm{O} 4-$ 1.8 v.u. Clearly, the $\mathrm{O} 3$ and $\mathrm{O} 4$ atoms are severely underbonded, indicating considerable tensile bond strain. At higher temperatures, the situation is similar apart from the weaker overbonding of the $\mathrm{Y} 1$ atoms. Fig. 10 displays a structural model at $850{ }^{\circ} \mathrm{C}$ with the ADPs drawn at $70 \%$ probability. The most pronounced anisotropy of the APDs is observed for the $\mathrm{Mn}, \mathrm{Y}$, and O3/O4 sites; similar anisotropy was obtained for all temperatures above $600{ }^{\circ} \mathrm{C}$. The Mn ADPs are strongly elongated normal to the mirror symmetry planes (i.e. $m$ and $c$ ), hinting at disordered local displacements of $\mathrm{Mn}$ toward one of the $\mathrm{O} 4$ atoms. The ADPs for the $\mathrm{O} 4$ and $\mathrm{Y} 2$ sites are strongly elongated along the $c$-axis, also suggesting some positional disorder for these atoms; likely, the displacements of $\mathrm{Mn}$ and $\mathrm{Y} 2$ are related via the metal-oxygen bonds in the $\mathrm{Mn}-\mathrm{O} 4-\mathrm{Y} 2$ links and are driven by the significant under-bonding of the $\mathrm{O} 4$ atoms. Overall, the underbonded $\mathrm{Mn}$ and $\mathrm{O} 3 / \mathrm{O} 4$ atoms are an important feature of the $\mathrm{YMnO}_{3}$ structure. Recently, Cheng et al. [21] reported that prolonged irradiation of $\mathrm{YMnO}_{3}$ by an electron beam in TEM results in formation of oxygen vacancies preferentially on the 03/04 sites. These results, which are supported by the density-functional calculations showing lower energies of formation for vacancies on the 03/O4 as opposed to 01/O2 sites [20], concur with our BVS assessments. The relatively weak $\mathrm{Mn}-\mathrm{O} 3 / \mathrm{O} 4$ bonds appear to play a role in the ability of $\mathrm{YMnO}_{3}$-based structures to absorb excess oxygen, as will be discussed in the next section.

The temperature dependence of the $\mathrm{Y} 1-\mathrm{O} 3$ bond length (obtained using the refinements with isotropic ADPs to minimize correlations among the structural variables) exhibits an anomaly between $850{ }^{\circ} \mathrm{C}$ and $600{ }^{\circ} \mathrm{C}$ with a marked shortening of this bond (on cooling) from $\approx 2.62 \AA$ to 
$\approx 2.32 \AA$ (Fig. 11). The trend for $\mathrm{Y} 2-\mathrm{O} 4$ bond length was less pronounced. We obtained similar results from the data collected on two independently synthesized $\mathrm{YMnO}_{3}$ samples measured on the BT-1 and Polaris instruments, respectively. Previous neutron diffraction studies of $\mathrm{YMnO}_{3}$ [7] reported abrupt shortening (on cooling) of the $\mathrm{Y} 1-\mathrm{O} 3$ bond around $670{ }^{\circ} \mathrm{C}$, which has been attributed to an isosymmetric phase transition. In the present case, the temperature range over which this shortening occurs spans $\approx 200{ }^{\circ} \mathrm{C}$. The sources of this discrepancy remain unclear. The shortening of the $\mathrm{Y} 1-\mathrm{O} 3$ bond length occurs concurrently with a decrease in the $\mathrm{Mn} a$-axis displacements towards the $\mathrm{O} 3$ oxygens; the latter effect results in abnormal under-bonding of the $\mathrm{O} 3$ atoms which is mitigated by forming a shorter $\mathrm{Y} 1-\mathrm{O} 3$ bond.

Fig. 12a compares the experimental room-temperature neutron PDF for $\mathrm{YMnO}_{3}$ with that calculated in the PDFGUI software using a Rietveld-refined model. For $r>10 \AA$, this model provides adequate description of the data. However, for shorter distances, the match is poor suggesting significant differences between the average and local structures. The agreement between the experimental and calculated PDFs can be improved markedly (Fig. 12b) by introducing a functional dependence of the PDF peak widths, $\sigma_{i j}$, on interatomic distance, $r_{\mathrm{ij}}$, as implemented in PDFGUI:

$$
\sigma_{i j}^{2}={\sigma^{\prime}}_{i j}^{2} \sqrt{\left(1-\Delta / r_{i j}\right)}
$$

where $\sigma^{\prime}{ }_{i j}$ is the peak width in case of uncorrelated atomic motion, which is computed from the ADPs of atoms $i$ and $j$, while the parameter $\Delta$ describes the effect of displacement correlations on $\sigma_{i j}$. The refinements yield $\Delta \approx 1.6$ (all other parameters were kept fixed at their Rietveld-derived values). This analysis suggests the existence of strong positive correlations among the atomic displacements with correlation lengths greater than $10 \AA$. Obviously, the model encoded in Equation 1, which describes all the correlations using a single global parameter $\Delta$, while illustrative, is over simplistic for a multi-element compound. A more detailed insight into the nature of displacement correlations and deviation of the local structure from the average was obtained using a large-box atomistic modeling of the total-scattering data in RMCProfile.

Simultaneous RMC fitting of the neutron PDF, $S(Q)$, and Bragg intensities yielded atomic configurations that described all the data satisfactorily (Fig. 13). The average $P 6_{3} \mathrm{~cm}$ symmetry, albeit not enforced, was preserved in the RMC-refined configurations and the coordinates of Wyckoff positions and their corresponding ADPs, which were deduced by averaging multiple refined configurations, were similar to those obtained from the Rietveld refinements. Consistent with the PDFGUI analysis, local atomic displacements were found to be strongly correlated. In particular, large positive correlations exist among the $\mathrm{Y}$ and $\mathrm{O}$ ( $\mathrm{O} 1$ and $\mathrm{O} 2$ in the crystallographic model) displacements within the layers of $\left[\mathrm{YO}_{7}\right]$ polyhedra; these correlations propagate over distances $>10 \AA$. Strong but shorter-range positive correlations were also identified among the local $\mathrm{Mn}$ and $\mathrm{O}$ displacements. Conceivably, such positive in-plane correlations reflect natural rigidity of the triangular lattice. Small negative (correlation 
parameter $\approx-0.1)$ correlations were observed for the $z$-axis displacements of the neighboring $Y$ and $\mathrm{O} 3 / \mathrm{O} 4$ atoms along the $\mathrm{Y}-\mathrm{O}-\mathrm{Y} c$-axis chains.

Overall, the local atomic coordinations were found to closely mimic those observed in the average structure. One exception is the coordination of $\mathrm{Mn}$. On average, $\mathrm{Mn}$ forms 2 short bonds $(\approx 1.87 \AA$ ) with the apical 01 and 02 atoms, and 3 longer bonds $(\approx 2.06 \AA, \approx 2.07 \AA$ ) with the equatorial $03(\approx 2.07 \AA)$ and $04(\approx 2.08 \AA)$ atoms. However, locally, $\mathrm{Mn}$ appears to be systematically off-centered within the 03-04-04 triangles with significant shortening (to $\approx 1.95$ $\AA$ ) of one of these bonds. Presumably, this local Mn off-centering mitigates the under-bonding of the $\mathrm{Mn}$ and $\mathrm{O3/O4}$ atoms. The RMC refinements suggest that at room temperature $\mathrm{Mn}$ is displaced randomly toward the $\mathrm{O} 3$ and $\mathrm{O} 4$ sites and the average mirror symmetry of the $\mathrm{Mn}$ probability density distribution is preserved.

Thus, the discrepancy between the experimental PDF and the one predicted from the Rietveldrefined average-structure model is readily accounted for by the correlated thermal motion. Atomic displacements obtained from RMC analyses typically represent a superposition of the low-energy thermal and static displacements. Apparently, in $\mathrm{YMnO}_{3}$ at room temperature, we see a strong prevalence of thermal displacements related to acoustic phonons. PDFGUI fits to the PDFs measured at elevated temperatures $\left(225^{\circ} \mathrm{C}, 600^{\circ} \mathrm{C}\right)$ demonstrate that the correlation parameter $\Delta$ decreases progressively with increasing temperature, which can be attributed to a normal increase in the contribution of optical phonons.

Degradation of the goodness-of-fit for Rietveld refinements for $\mathrm{T}<400{ }^{\circ} \mathrm{C}$ (Fig. 9) can be associated with an uptake of the interstitial oxygen at these temperatures. As we show below, these excess oxygen atoms strongly affect the $\mathrm{YMnO}_{3}$ structure so that even their sub-percent concentrations are likely to produce measurable effects on the diffracted intensities.

Structure of Ti-substituted $\mathrm{YMnO}_{3}$ : Accommodation of Excess Oxygen

For $\mathrm{YMn}_{0.875} \mathrm{Ti}_{0.125} \mathrm{O}_{3+\delta}$, both the $P \sigma_{3} \mathrm{~cm}$ and $P \sigma_{3} / \mathrm{mmc}$ models with isotropic ADPs provide inadequate fit to the room temperature NPD data. The quality of the fit improves considerably once the anisotropic ADPs are introduced. The refined model features strongly anisotropic ADPs for the $Y$ and $\mathrm{O} 4$ sites with abnormally large $U_{33}$ components. However, even with the anisotropic ADPs, the quality of the fit remains poor.

The excess oxygen atoms $\left(\mathrm{O}_{1}\right)$ can be accommodated in large interstices having their centers located on 3-fold symmetry axes (Wyckoff positions $6 c,\{x 0 z\}, x \approx 2 / 3, z \approx 0$ ) (Fig. 14) [12]. The distances from these sites to the cations and host oxygen $\left(\mathrm{O}_{\mathrm{H}}\right)$ atoms $\mathrm{O} 1$ and $\mathrm{O} 2$ are sound; however, the distances to $\mathrm{O} 3$ and $\mathrm{O} 4$, residing at $2 a$ and $4 b$ Wyckoff positions are unphysically short $(\approx 2 \AA)$. We constructed a model with $13 \%$ of the interstitial sites occupied by the excess oxygen atoms and refined it using an RMC method. The actual distribution of $\mathrm{O}_{1}$ is likely to be non-random, but as a zero approximation we ignored these details. The neutron pair- 
distribution function and Bragg profile were fitted simultaneously under a restraint imposed on the shortest distances between the $\mathrm{O}_{\mathrm{I}}$ and $\mathrm{O}_{\mathrm{H}}$ atoms to avoid unphysical configurations. We performed these refinements for the two datasets collected on Polaris at $\mathrm{T}=300{ }^{\circ} \mathrm{C}$ : one on the $1^{\text {st }}$ heating (structure with excess oxygen) and another on the $2^{\text {nd }}$ heating in vacuum (structure without excess oxygen) (Fig. 15). In both cases, a satisfactory agreement between the experimental and calculated data was obtained (Fig. 16).

Fig. 17 presents key partial pair-distribution functions for the structure with excess oxygen. All the distance distributions appear to be sensible. The $O_{1}$ atoms are bonded to $3 \mathrm{Mn}$ with the

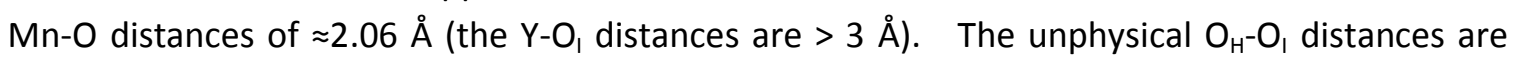
avoided by anti-parallel (correlation parameter $\approx-0.3$ ) displacements of the nearest-neighbor $\mathrm{O}_{\mathrm{H}}$ $(03,04)$ and $\mathrm{O}_{1}$ atoms both along the $c$-axis $\left(\mathrm{O}_{\mathrm{H}}-\approx 0.27 \AA \mathrm{O}_{1}-\approx 0.17 \AA\right)$ and in the $a b$ plane $\left(\mathrm{O}_{\mathrm{H}}\right.$ - $\approx 0.4 \AA ; \mathrm{O}_{1}-\approx 0.14 \AA$ ). For $\delta=0.13$, most of the $\mathrm{Mn}$ atoms that have $\mathrm{O}_{\text {। }}$ as their nearest neighbors are 6-fold coordinated by oxygen; the bases of the resulting oxygen polyhedra are highly distorted polygons. On average, the $\mathrm{O} 3$ and $\mathrm{O} 4$ atoms still occupy high-symmetry $2 a(0$, $0, z \approx 0.5)$ and $4 b(1 / 3,2 / 3, z \approx 0)$ Wyckoff positions. However, those 03 and 04 atoms that acquire $\mathrm{O}_{1}$ as nearest neighbors are offset so that their probability density at the average locations is zero (Fig. 18). In contrast, the probability density for the remaining $\mathrm{O} 3$ and $\mathrm{O} 4$ atoms that have no $O_{1}$ neighbors is maximal at the $2 a$ and $4 b$ sites, respectively (Fig. 18). The $c$ axis displacements of $\mathrm{O} 3$ and $\mathrm{O} 4$ are strongly correlated with those of $\mathrm{Y}$ (correlation parameter $\approx 0.5$ ), as dictated by the bonding requirements of the $Y$ atoms; these displacements account for the abnormally large $U_{33}$ values of $Y$ and $O 4$ identified in the Rietveld refinements. The probability density distributions for the $Y$ atoms feature double sites split (by $\approx 0.43 \AA$ ) along the $c$-axis (Fig. 18); the disorder of $Y$ over the split sites destroys the $P \sigma_{3} \mathrm{Cm}$ superstructure with concurrent disappearance of the corresponding extra reflections in the samples containing excess oxygen. The BVS of $\mathrm{Mn}$ in this structure increases to an ideal value of $3.0 \mathrm{v} . \mathrm{u}$., although the $\mathrm{O} 3$ and $\mathrm{O} 4$ atoms still remain severely underbonded (1.6 v.u.).

Once the extra oxygen atoms are desorbed (e.g., by heating and cooling in vacuum), the superstructure is restored. Rietveld refinements for the $\mathrm{YMn}_{0.875} \mathrm{Ti}_{0.125} \mathrm{O}_{3}$ sample in the "stoichiometric" (i.e. all $\mathrm{Ti}^{3+}$ ) state at $300{ }^{\circ} \mathrm{C}$ yield structural parameters and thermal ellipsoids similar to those obtained for $\mathrm{YMnO}_{3}$ at a similar temperature $\left(275^{\circ} \mathrm{C}\right)$. A corresponding PDF can be adequately described using the Rietveld-refined model with $\Delta=0.65$, which is similar to a value of $\Delta$ obtained for $\mathrm{YMnO}_{3}$ at this temperature. The BVS of $\mathrm{Mn}$ (calculated ignoring the effect of Ti substitution, which is unlikely to significantly affect the results) in this vacuumtreated $\mathrm{YMn}_{0.875} \mathrm{Ti}_{0.125} \mathrm{O}_{3}$ is about $2.7 \mathrm{v}$.u, whereas the BVS of $\mathrm{Y} 1$ and $\mathrm{Y} 2$ is $3.16 \mathrm{v} . \mathrm{u}$. and $3.02 \mathrm{v} . \mathrm{u}$., respectively. Conceivably, the under-bonding of $\mathrm{Mn}$, which in $\mathrm{YMn}_{0.875} \mathrm{Ti}_{0.125} \mathrm{O}_{3}$ appears to be even stronger than in $\mathrm{YMnO}_{3}$, promotes absorption of interstitial oxygen in this structural type. The relatively weakly bonded $\mathrm{O} 3$ and $\mathrm{O} 4$ atoms can undergo large displacements, which as described in the preceding paragraph, are needed for structural accommodation of the excess oxygen. 
Even after the excess oxygen atoms have been supposedly removed, the $c$-lattice parameter in the Ti-substituted samples still varies anomalously with temperature (Fig. 6). The $c(T)$ trends derived from the XRD data for both $\mathrm{x}=0.05$ and $\mathrm{x}=0.125$ are non-monotonic with a minimum at $\approx 500{ }^{\circ} \mathrm{C}$ (i.e. higher that the temperature of the redox reaction), which may point to a genuine structural anomaly. However, a $c(T)$ dependence determined for the "stoichiometric" $x=0.125$ sample (Fig. 7) in the neutron experiment is different, even though some anomaly at $500{ }^{\circ} \mathrm{C}$ still can be observed. Such a discrepancy between the trends obtained under different environmental conditions suggest residual redox effects. Unfortunately, the temperature range probed in the neutron experiment is relatively narrow, limiting the comparison. In any case, Rietveld refinements using both the XRD and neutron diffraction data did not reveal any clear structural changes that could be reliably identified with the anomaly in $c(T)$. In particular, no characteristic changes in the $\mathrm{Y} 1-\mathrm{O} 3 / \mathrm{Y} 2-\mathrm{O} 4$ bond lengths, of the kind seen in $\mathrm{YMnO}_{3}$ around 600 ${ }^{\circ} \mathrm{C}$, were observed.

Interestingly, in contrast to the paramagnetic $\mathrm{YMnO}_{3}$, the $x=0.125$ sample exhibited magnetic hysteresis near room temperature, with remnant mass magnetization $\approx 0.005 \mathrm{Am}^{2} / \mathrm{kg}$ and coercive field $\left(H_{c}\right)>4 \mathrm{kA} / \mathrm{m}$ after removal of the paramagnetic background (Fig. 20a, b). Both the ferromagnetic and the paramagnetic response weakened upon heating (Fig. 20). Similar hysteresis loops were obtained immediately before and after heating the sample to $677^{\circ} \mathrm{C}(950$ $\mathrm{K}$ ) in vacuum, perhaps due to the short duration of the measurement at high temperatures. In contrast, when the hysteresis loops were repeated after the sample was stored in air for 6 months, the magnetic response was substantially different. Specifically, the $350 \mathrm{~K}$ coercivity more than doubled from $4 \mathrm{kA} / \mathrm{m}$ initially to $8.8 \mathrm{kA} / \mathrm{m}$ after extended exposure to air. Furthermore, while the coercivity monotonically decreased upon heating in both cases, it comfortably exceeded the flux trappage in the superconducting magnet only for the sample with extended exposure to air $\left(\mathrm{H}_{\mathrm{c}} \approx 3.2 \mathrm{kA} / \mathrm{m}\right.$ at $\left.677{ }^{\circ} \mathrm{C}(950 \mathrm{~K})\right)$. The paramagnetic response also decreased by $25 \%$ after extended exposure to air. These differences can be related to the slow change in the structure and oxidation state with time and exposure to oxygen. In addition, the magnetization $(M)$ of the $x=0.125$ sample measured during its first heating in vacuum under a magnetic field of $80 \mathrm{kA} / \mathrm{m}$ decreased with increasing temperature and exhibited an anomaly at $\mathrm{T} \approx 477^{\circ} \mathrm{C}(750 \mathrm{~K})$, which can be related to the redox reactions (Fig. 20c). Cooling this sample in vacuum to room temperature resulted in a reduction of $\approx 12 \%$ in the magnetization. Subsequent thermal cycling produced $M(T)$ curves similar to those obtained on cooling after the first heating. For $\mathrm{YMnO}_{3}$, the $\mathrm{M}(\mathrm{T})$ curves exhibited no anomalies and were retraceable during thermal cycling, similar to the TGA data. Elucidating the origins of the observed magnetic behavior of Ti-substituted $\mathrm{YMnO}_{3}$ requires a separate study.

\section{Conclusions}

$\mathrm{YMnO}_{3}$ remains approximately stoichiometric in flowing air and helium at least up to $1150{ }^{\circ} \mathrm{C}$. Its local structure is well represented by the average $P \sigma_{3} \mathrm{~cm}$ model except for the coordination of 
the $\mathrm{Mn}$ atoms which are locally displaced off the mirror planes. An apparent discrepancy between the experimental atomic pair-distribution function and that calculated using the Rietveld-derived average-structure model arises from the strong, positive nanoscale-range correlations among the thermal atomic displacements. Substitution of $\mathrm{Ti}$ into $\mathrm{YMnO}_{3}$ results in significant absorption of oxygen from air below $400{ }^{\circ} \mathrm{C}$. This absorption is attributed to the oxidation reactions $\mathrm{Ti}^{3+} \rightarrow \mathrm{Ti}^{4+}$ and $\mathrm{Mn}^{3+} \rightarrow \mathrm{Mn}^{4+}$, yielding an equilibrium formula $\mathrm{YMn}^{3+}{ }_{0.875-x-}$

${ }_{\mathrm{y}} \mathrm{Mnn}^{4+}{ }_{\mathrm{y}} \mathrm{Ti}^{4+}{ }_{\mathrm{x}} \mathrm{O}_{3+\delta}$. For the composition $x=0.125$, which was studied in detail, we estimated $\mathrm{y} \approx 0.14$ and $\delta \approx 0.13$. The $\mathrm{Ti}^{3+} \leftrightarrow \mathrm{Ti}^{4+}$ and $\mathrm{Mn}^{3+} \leftrightarrow \mathrm{M}^{4+}$ reactions are reversible and occur around $\approx 400{ }^{\circ} \mathrm{C}$ and $\approx 250^{\circ} \mathrm{C}$, respectively. Both reactions exhibit relatively sluggish kinetics, which are sensitive to sample-processing conditions. The excess oxygen atoms are accommodated in the large interstices of the $\mathrm{YMnO}_{3}$ structure. Bond valence sum calculations suggest that oxygen absorption is driven by the under-bonding of $\mathrm{Mn} / \mathrm{Ti}$. Chemical-bond strain associated with the interstitial oxygen is effectively relieved by the locally correlated displacements of the host atoms, which provide reasonable bond lengths among all the species. A key role in this relaxation is played by the significantly underbonded host oxygen atoms that form the trigonal bases of the $\left[\mathrm{MnO}_{5}\right]$ bi-prisms. The presence of Ti enhances the $\mathrm{Mn}^{3+} \rightarrow \mathrm{Mn}^{4+}$ reaction, which, remarkably, proceeds even at ambient conditions, accounting for a large fraction of excess oxygen in the samples as they approach equilibrium. Our results suggest that Ti substitution can be useful for optimizing the oxygen absorption properties of $\mathrm{RMnO}_{3}$ compounds and as such warrants further investigation.

\section{Acknowledgements}

Portions of this research were carried out at the National Synchrotron Light Source (NIST beamline X23A2), Brookhaven National Laboratory, supported by the U.S. Department of Energy, Office of Science, Office of Basic Energy Sciences, under Contract No. DE-AC0298CH10886. We thank the STFC (UK) for access to the ISIS facility. The neutron total-scattering data for pure $\mathrm{YMnO}_{3}$ were collected as a part of Prof. Martin Dove's beam time (proposal RB1410591) on the Polaris diffractometer at ISIS; a full analysis of these data will be published in a further article. 


\section{Figure Captions}

Fig. 1: Crystal structure of hexagonal $\mathrm{YMnO}_{3}$ structure (space group $P 6_{3} \mathrm{~cm}$ ). The $\mathrm{Mn}$ - and $\mathrm{Y}$ centered polyhedra are indicated using green and blue colors, respectively.

Fig. 2: TGA curves for (a) $\mathrm{YMnO}_{3}$ in air (blue) and oxygen (red) and (b) $\mathrm{YMn}_{0.875} \mathrm{Ti}_{0.125} \mathrm{O}_{3+\delta}$ in air; cycles 3 and higher were essentially superimposable on cycle 2 (red), but the unique first cycle (blue) is again observed if the residue is fully re-oxidized.

Fig. 3: TGA curves for as-prepared $\mathrm{YMn}_{0.875} \mathrm{Ti}_{0.125} \mathrm{O}_{3+\delta}$ sample in (a) helium (Cycles 1 and 2) and then in (b) air. Fig. 3b includes the DSC curve (orange).

Fig. 4: (a) Variable-temperature $\mathrm{X}$-ray absorption spectra of the $\mathrm{Mn} K$-edge in the as-prepared sample of $\mathrm{YMn}_{0.875} \mathrm{Ti}_{0.125} \mathrm{O}_{3+\delta}$. (b) Magnified view of the main absorption edge revealing a shift to higher energies on heating between $200^{\circ} \mathrm{C}$ to $300{ }^{\circ} \mathrm{C}$.

Fig. 5: Variable-temperature XRD patterns collected during the (a) first and (b) second heating of the as-prepared sample of $\mathrm{YMn}_{0.875} \mathrm{Ti}_{0.125} \mathrm{O}_{3+\delta}$ in air. The superlattice reflections are indicated using arrows. The curves are labelled with temperatures in ${ }^{\circ} \mathrm{C}$.

Fig. 6: Temperature dependences of the lattice parameters derived using XRD for the $\mathrm{YMn}_{1}$ ${ }_{x} \mathrm{Ti}_{\mathrm{X}} \mathrm{O}_{3}$ samples heated (second heating) in air (red) and helium (blue). The first heating was performed in the same atmosphere. (a, b) $\mathrm{YMnO}_{3}$, (c, d) $\mathrm{YMn}_{0.95} \mathrm{Ti}_{0.05} \mathrm{O}_{3+\delta}$, (e, f) $\mathrm{YMn}_{0.875} \mathrm{Ti}_{0.125} \mathrm{O}_{3+\delta}$. The errors bars fit within the symbol size.

Fig. 7: Temperature dependence of the lattice parameters of $\mathrm{YMn}_{0.875} \mathrm{Ti}_{0.125} \mathrm{O}_{3+\delta}$ derived from the neutron diffraction data (Polaris). Blue $-1^{\text {st }}$ heating of the as-prepared sample under 400 mTorr helium. Red $-2^{\text {nd }}$ heating of the same sample in vacuum. The inset shows a magnified view of the dependence of the $c$-parameter upon the $2^{\text {nd }}$ heating.

Fig. 8: Experimental (red) and calculated (blue) neutron diffraction patterns of $\mathrm{YMnO}_{3}$ at room temperature. The residual is shown in green. The calculated pattern corresponds to the $\mathrm{P}_{3} \mathrm{Cm}$ model.

Fig. 9: Goodness of fit, $\chi^{2}$, for the Rietveld refinements of $\mathrm{YMnO}_{3}$ using variable-temperature neutron powder diffraction data collected on BT-1.

Fig. 10: A rendering of the $\mathrm{YMnO}_{3}$ structure at $700{ }^{\circ} \mathrm{C}$ obtained from Rietveld refinements using the neutron diffraction data (BT-1). The thermal ellipsoids are plotted at $70 \%$ probability. Similar anisotropy of the ADPs was obtained for all $T>600{ }^{\circ} \mathrm{C}$. (top) A side view of the structure, (bottom) a top view of a single $\left[\mathrm{MnO}_{3}\right]$ layer.

Fig. 11: Temperature dependence of the (a) $\mathrm{Y} 1-\mathrm{O} 3$ and (b) $\mathrm{Mn}-\mathrm{O} 3$ bond lengths in $\mathrm{YMnO}_{3}$ (BT-1 data). The error bars represent 1 estimated standard deviation as calculated by GSAS. The actual errors in the $\mathrm{Mn}-\mathrm{O}$ bond length appear to be larger. 
Fig. 12: (a) Experimental and calculated neutron PDF for $\mathrm{YMnO}_{3}$. The calculated PDF corresponds to the Rietveld-refined structural model. (b) A fit of the experimental PDF in PDFGUI using the same model (with all the parameters fixed at their Rietveld-derived values) and a single variable $\Delta$ introduced to account for correlations among the atomic displacements.

Fig. 13: Results of the RMC fit of the neutron PDF (a) and Bragg profile (b) measured at room temperature for $\mathrm{YMnO}_{3}$.

Fig. 14: A rendering of atomic arrangements around the large interstices that accommodate the interstitial oxygen atoms (black sphere, $\mathrm{O}_{1}$ ). The arrows illustrate the correlated displacements of the interstitial and host atoms.

Fig. 15: Neutron diffraction patterns (a) and PDFs (b) for the $\mathrm{YMn}_{0.875} \mathrm{Ti}_{0.125} \mathrm{O}_{3}$ sample at $300 \mathrm{~K}$ recorded (red) during the first heating of the as-prepared sample under $400 \mathrm{mTorr}$ He and (blue) during the second heating in vacuum.

Fig. 16: Results of the RMC fit of the neutron PDF (a, b) and Bragg profile (c, d) measured at 300 ${ }^{\circ} \mathrm{C}$ for the as-prepared $\mathrm{YMn}_{0.875} \mathrm{Ti}_{0.125} \mathrm{O}_{3}$ sample upon its $(\mathrm{a}, \mathrm{c})$ first heating in $400 \mathrm{mTorr}$ helium and $(b, d)$ subsequent heating in vacuum.

Fig. 17: Partial PDFs involving the interstitial oxygen atoms obtained using the RMC fits shown in Fig. $16(a, c)$.

Fig. 18: Probability density distributions projected onto the $x y$-plane for the host oxygen atoms that do (a) and don't have (b) interstitial oxygen atoms as their nearest neighbors.

Fig. 19: A projected probability density distribution for the $Y$ atoms along the z-direction in the structure with (a) and without (b) excess oxygen. (c) A distribution of the number of $Y$ atoms over $z$ for (a).

Fig. 20: (a) Dependence of magnetization on magnetic field for $\mathrm{YMn}_{0.875} \mathrm{Ti}_{0.125} \mathrm{O}_{3+\delta}$ at $350 \mathrm{~K}$ (red), $800 \mathrm{~K}$ (blue), and $950 \mathrm{~K}$ (green). (b) A magnified view of the low-field portion of (a). Ferromagnetic behavior is observed at all temperatures with the largest response seen at $350 \mathrm{~K}$. (c) Temperature dependence of magnetization for $\mathrm{YMn}_{0.875} \mathrm{Ti}_{0.125} \mathrm{O}_{3+\delta}$ measured under a magnetic field $\mathrm{H}=80 \mathrm{kA} / \mathrm{m}$. Red and blue curves correspond to the $1^{\text {st }}$ and $2^{\text {nd }}$ heating cycles, respectively. The blue curve retraces a cooling portion of the red curve. 


\section{References}

1. B. B. Van Aken, A. Meetsma, T. T. M. Palstra, Acta Cryst. C, 57, 230-232 (2001)

2. B. B. Van Aken, T. T. M. Palstra, A. Filippetti, N. A. Spaldin, Nature Mater., 3 [3], 164-170 (2004)

3. T. Choi, Y. Horibe, H. T. Yi, Y. J. Choi, W. D. Wu, S. W. Cheong, Nature Mater., 9 [3], $253-$ 258 (2010)

4. H. Das, A. L. Wysocki, Y. N. Geng, C. G. Fennie, Nature Comm., 5, 2998 (2014)

5. D. Y. Cho, J. Y. Kim, B. G. Park, K. J. Rho, J. H. Park, H. J. Noh, B. J. Kim, S. J. Oh, H. M. Park, et al., Phys. Rev. Lett., 98 [21], 217601 (2007)

6. C. J. Fennie and K. M. Rabe, Phys. Rev. B., 72 [10] 100103 (2005)

7. A. S. Gibbs, K. S. Knight, P. Lightfoot, Phys. Rev. B., 83 [9]. 094111 (2011)

8. M. Lilienblum, T. Lottermoser, S. Manz, S. M. Selbach, A. Cano, M. Fiebig, Nature Phys., $11[12], 1070(2015)$

9. M. Tomczyk, P. M. Vilarinho, A. Moreira, A. Almeida, J. Appl. Phys., 110 [6] 064116

10. P. R. Ren, H. Q. Fan, X. Wang, Appl. Phys. Lett., 103 [15], 152905 (2013)

11. S. Remsen, B. Dabrowski, Chem. Mater., 23 [17] 3818-3827 (2011)

12. C. Abughayada, B. Dabrowski, S. Kolesnik, D. E. Brown, O. Chmaissem, Chem. Mater., 27 [18], 6259-6267 (2015)

13. S. M. Selbach, A. N. Løvik, K. Bergum, J. R. Tolchard, M-A. Einarsrud, T. Grande, J. Solid State Chem., 196, 528-535 (2012)

14. M. Tomczyk, A. M. O. R. Semos, I. M. Reaney, P. M. Vilarinho, Scripta Mater., 67 [5], $427-430$ (2012) 
15. The identification of any commercial product or trade name does not imply endorsement or recommendation by the National Institute of Standards and Technology.

16. While the helium and vacuum atmospheres both feature low oxygen partial pressures, their effects on the redox behavior can be different.

17. B. Ravel and M. Newville, J. Synch. Rad. 12 [4], 537-541 (2005)

18. C. L. Farrow, P. Juhas, J. W. Liu, D. Bryndin, E. S. Bozin, J. Bloch, T. Proffen, S. J. L. Billinge, J. Phys. Cond. Matter., 19 [33] 335219 (2007)

19. M. G. Tucker, D. A. Keen, M. T. Dove, A. L. Goodwin, Q. Hui, J. Phys. Cond. Matter., 19 [33], 335218

20. I. D. Brown and D. Altermatt, Acta Cryst. B., 41, 244-247 (1985)

21. S. Cheng, M. Li, Q. Meng, W. Duan, Y. G. Zhao, X. F. Sun, Y. Zhu, J. Zhu, Phys. Rev. B., 93, 054409 (2016) 


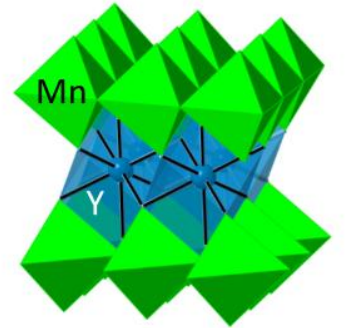



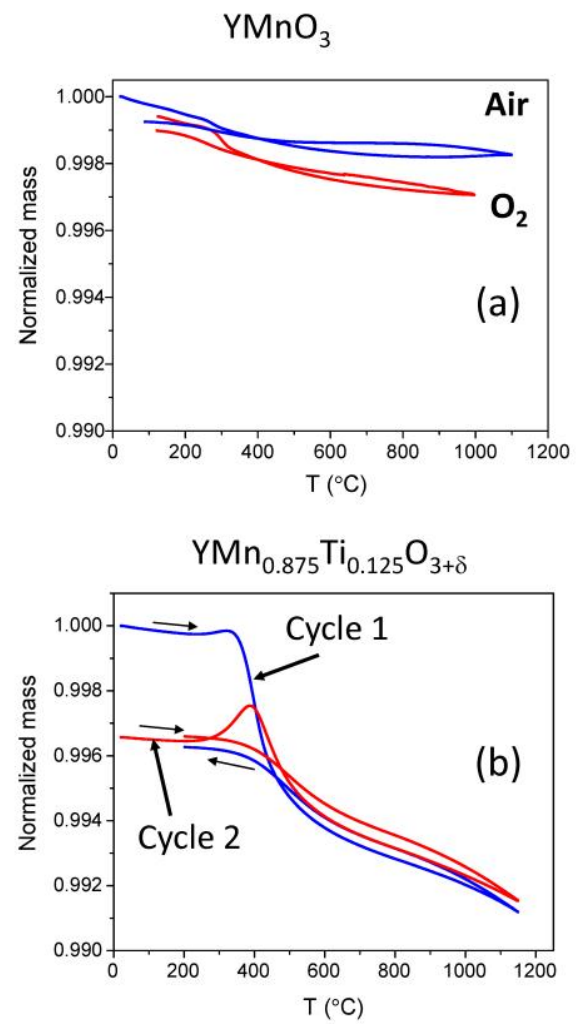

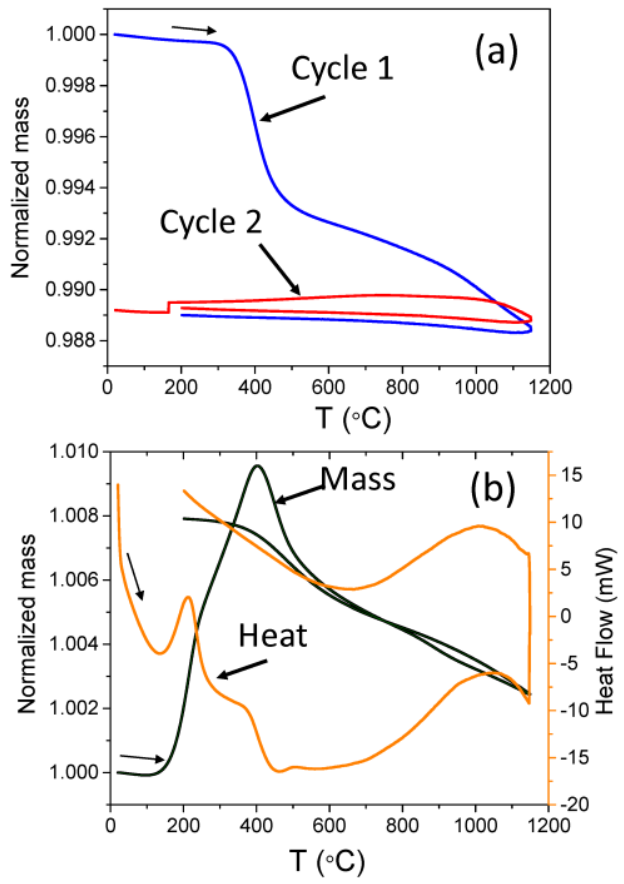

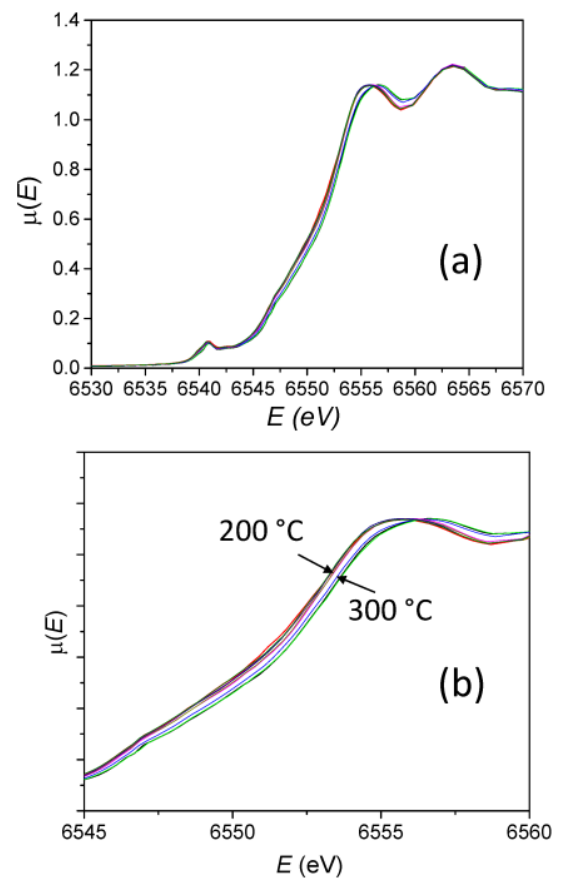

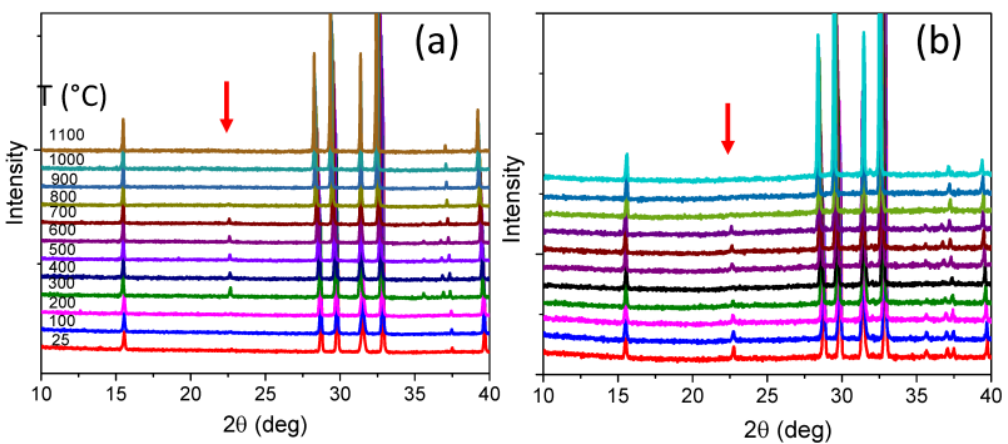

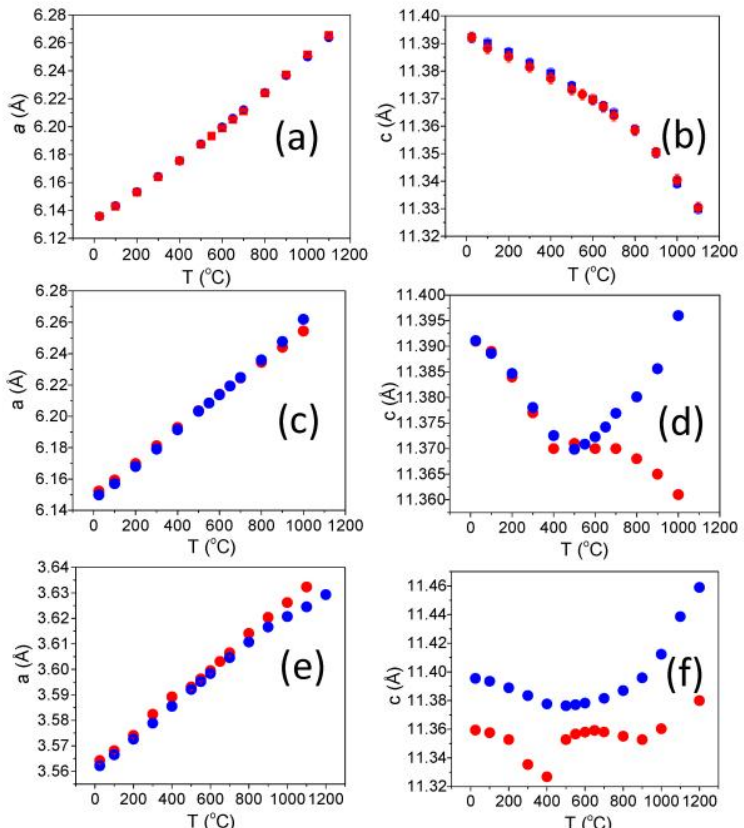

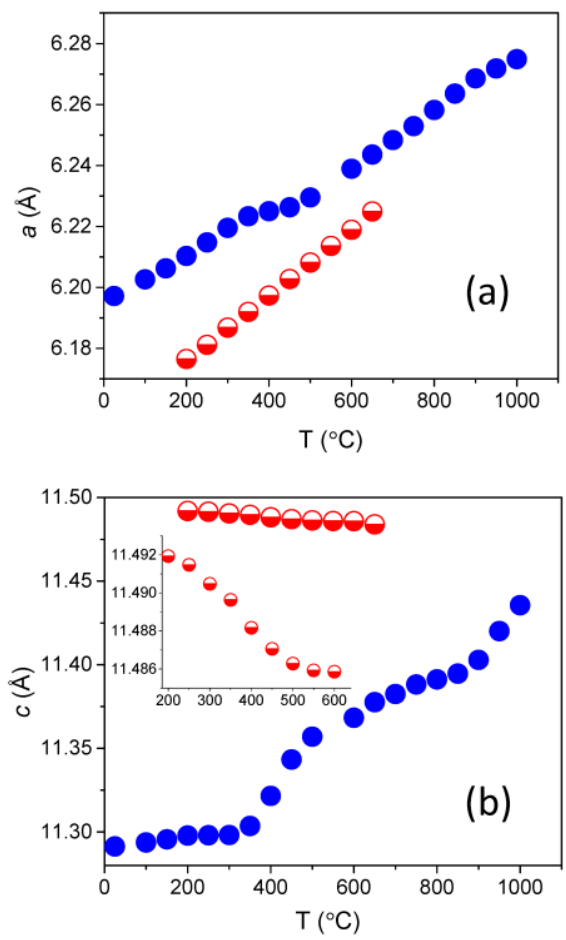

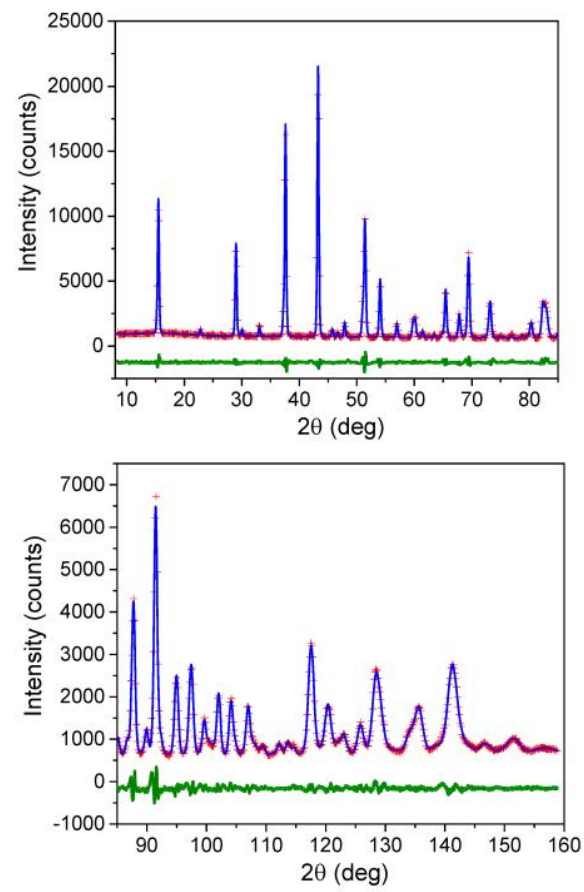


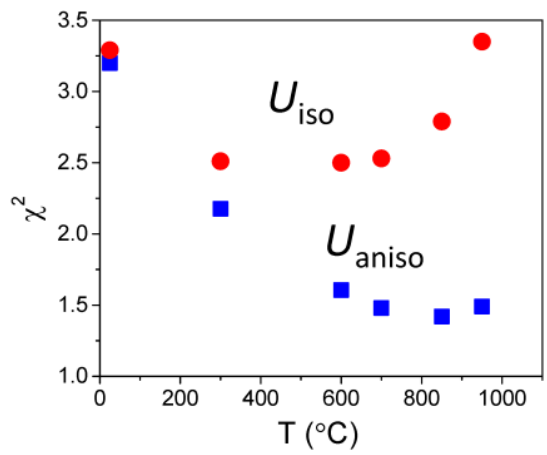




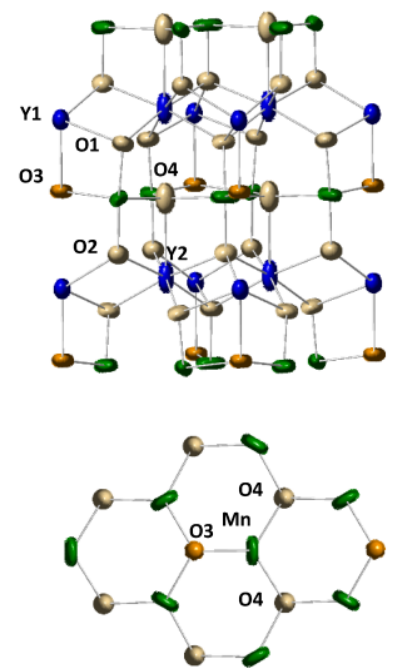



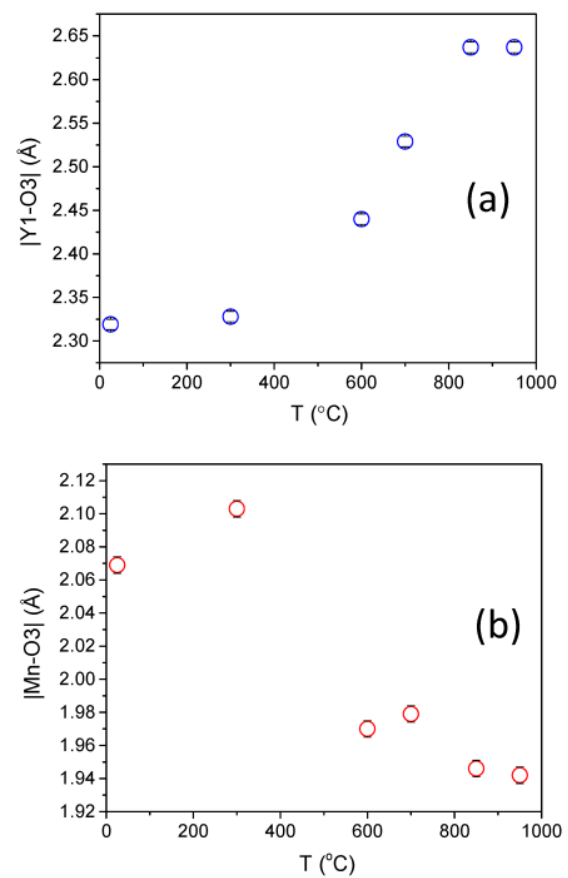

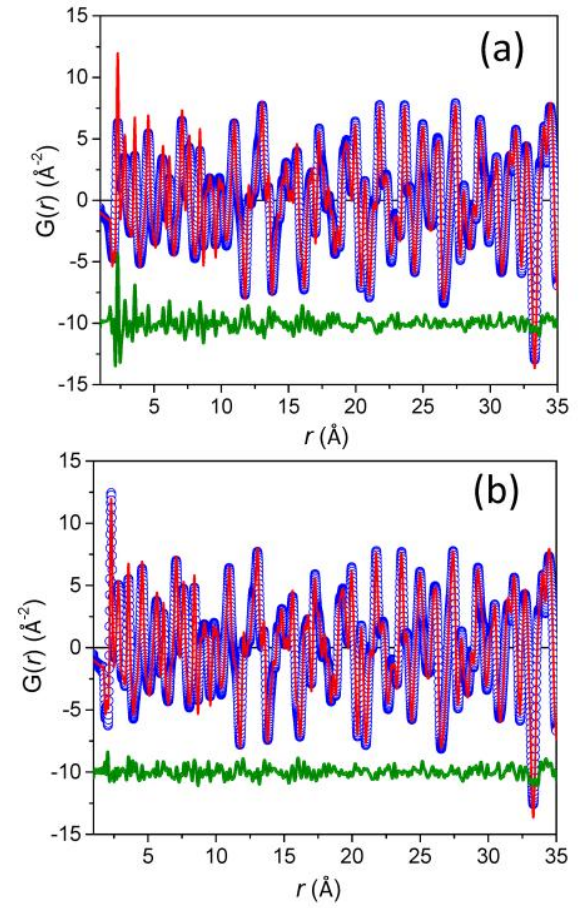

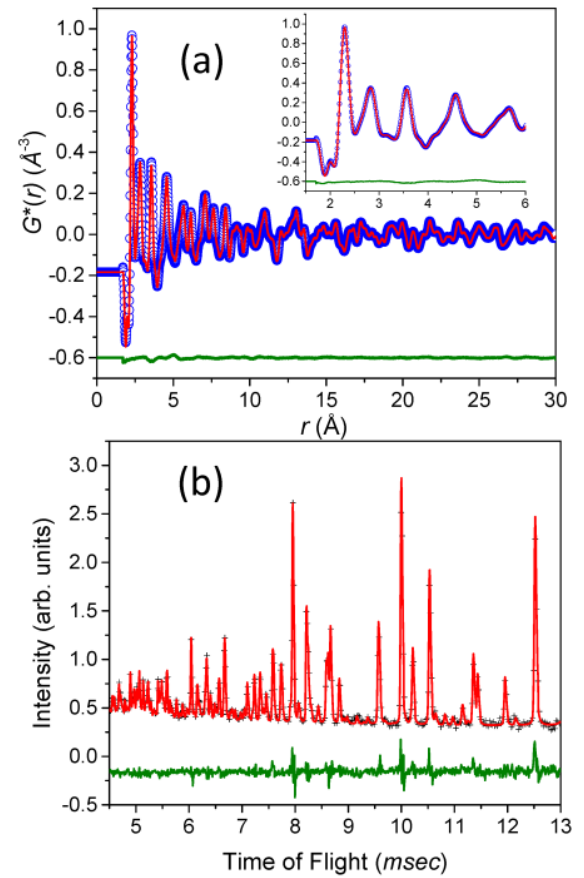


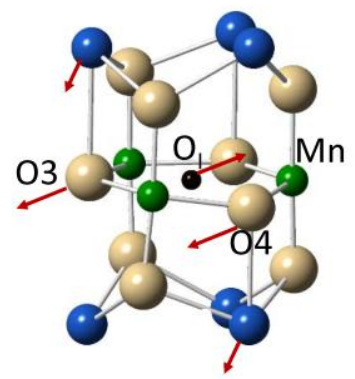



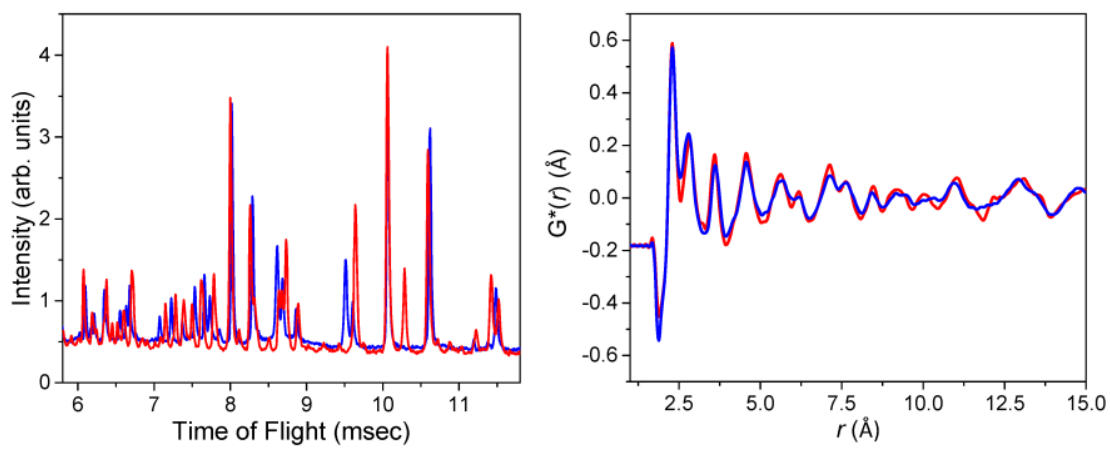

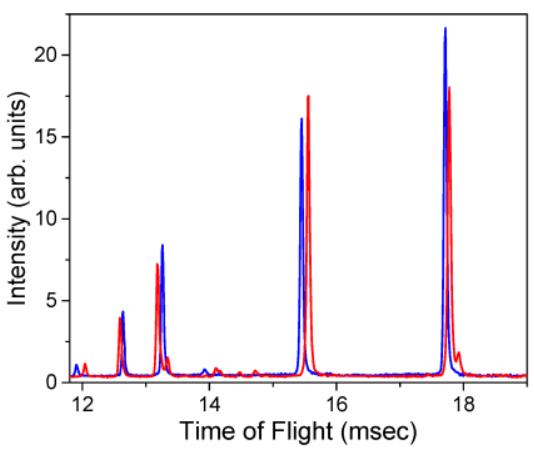

(a)

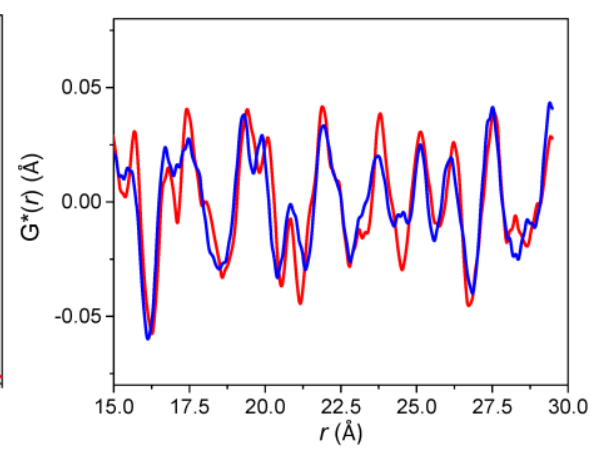

(b) 

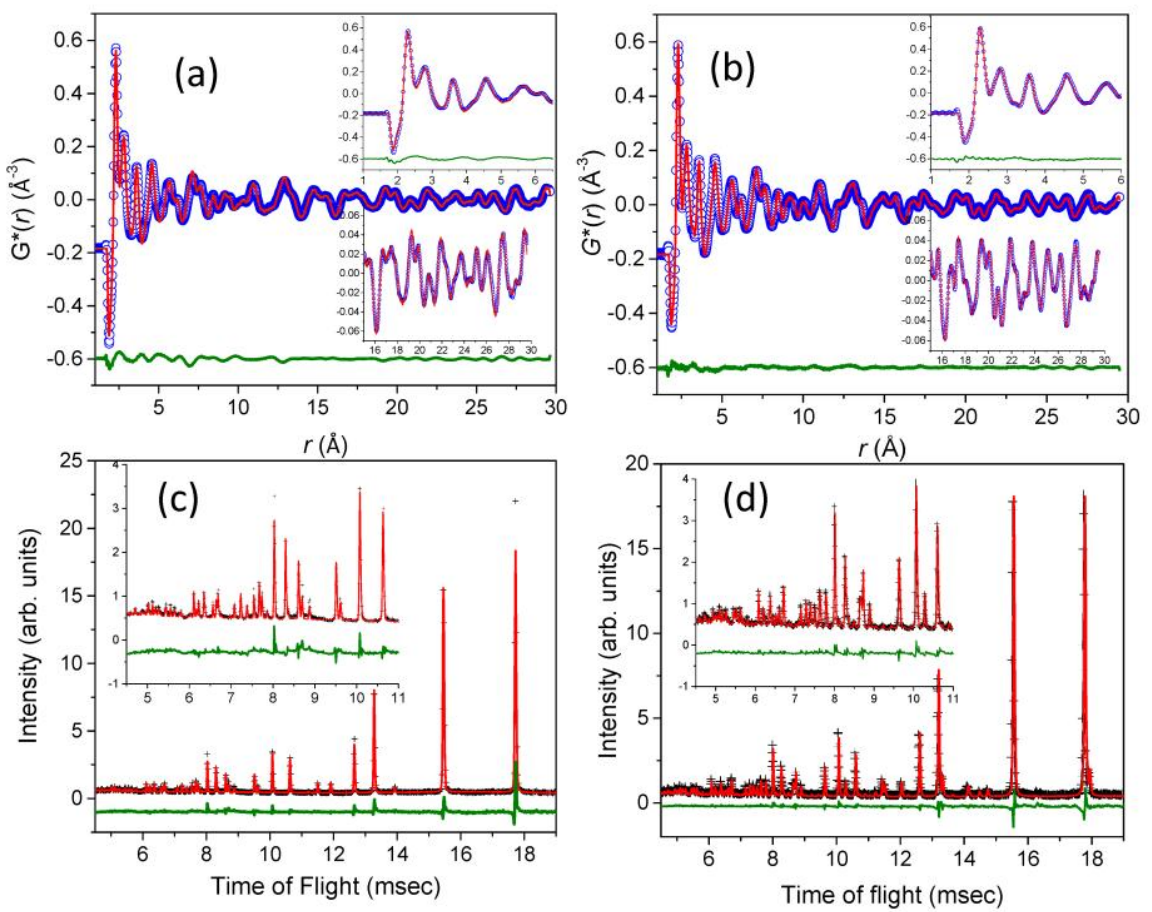


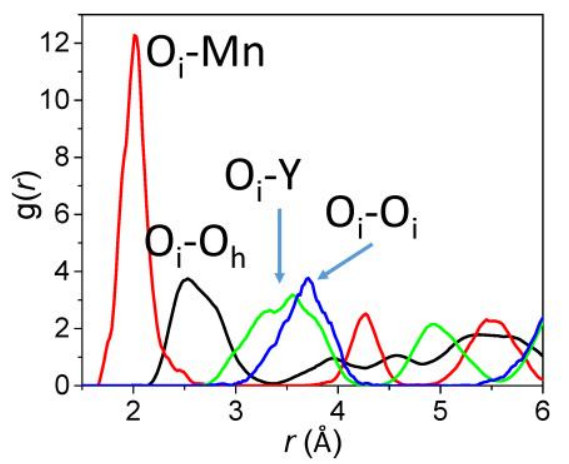



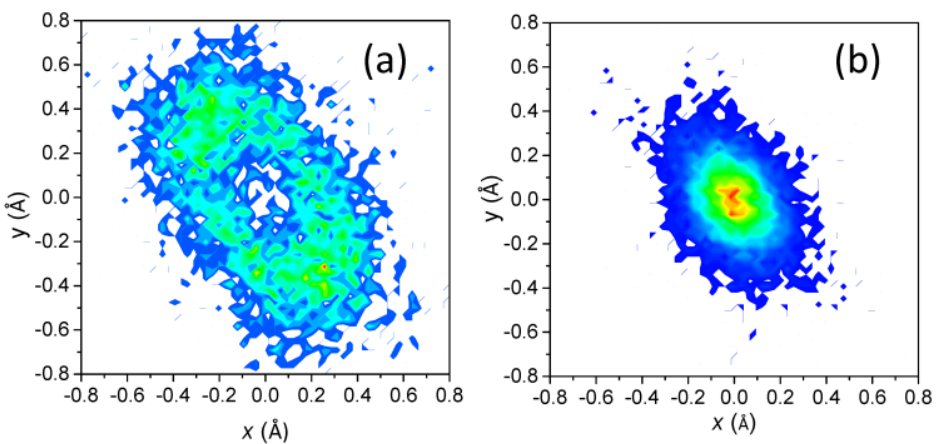

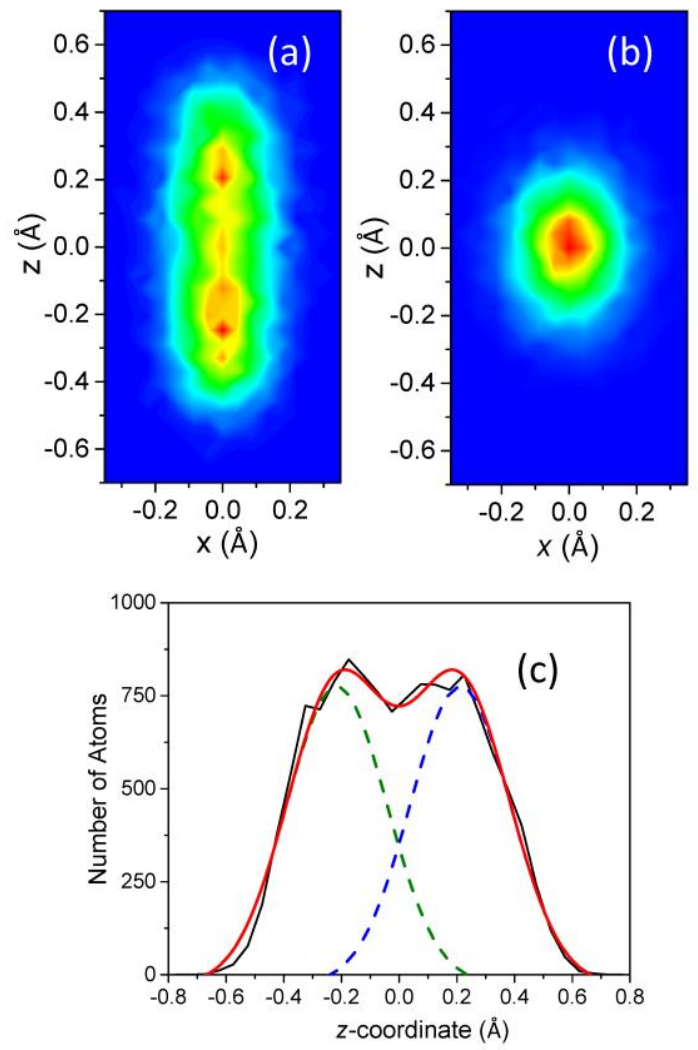

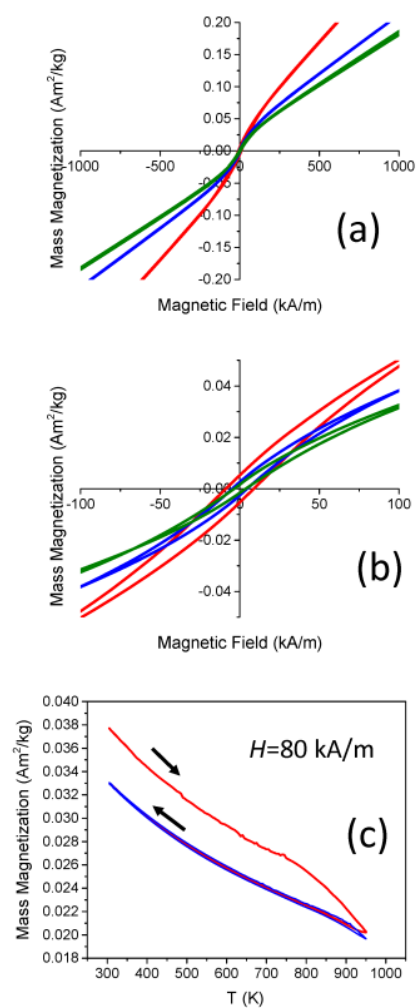


\section{Abstract figure caption:}

Concurrent redox reactions involving Ti and Mn yield facile absorption/desorption of excess oxygen. 
$1 / 2 O_{2}($ gas $)+2 M n^{3+} \leftrightarrow O_{i}^{\prime \prime}+2 M n^{4+}$

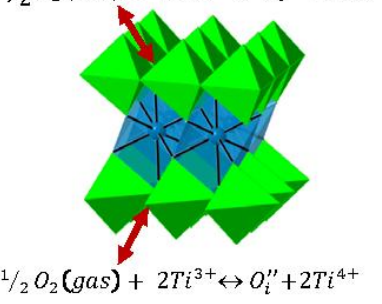

